\title{
Monte-Carlo Modeling of Cyclotron Resonant Scattering Features
}

\author{
Fritz-Walter Schwarm $^{* a b}$, G. Schönherr ${ }^{c}$, J. Wilms ${ }^{a b}$, P. Kretschmar ${ }^{d}$ \\ ${ }^{a}$ Dr. Karl Remeis-Sternwarte Bamberg \\ Sternwartstrasse 7, 96049 Bamberg, Germany \\ ${ }^{b}$ Erlangen Centre for Astroparticle Physics (ECAP) \\ Erwin-Rommel-Str. 1, 91058 Erlangen, Germany \\ ${ }^{c}$ Leibniz-Institut für Astrophysik Potsdam \\ An der Sternwarte 16, 14482 Potsdam, Germany \\ ${ }^{d}$ European Space Astronomy Centre (ESA/ESAC), Science Operations Department \\ Villanueva de la Cañada (Madrid), Spain \\ Email: fritz.schwarmesternwarte.uni-erlangen.de
}

\begin{abstract}
Cyclotron resonant scattering features (CRSFs) exhibit the fundamental physical processes in the line-forming region of highly magnetized neutron stars and allow for a direct measurement of a neutron star's magnetic field. The multiplicity of CRSFs seen in many sources - with 4U 0115+63 being the record holder showing a fundamental line plus 4 harmonics - and the complex shape of the fundamental line can provide unique insights into the physical properties and B-field geometry of the accretion column. The strong coupling of a photon's angle and energy due to relativistic effects are leading to a coupling of the seed photons spectral distribution and the accretion geometry and therefore hinder comprehension. The model presented here is using a Monte-Carlo approach to the problem by propagating individual photons through different kinds of cyclotron resonant scattering media. We produce tables of the simulated data allowing the fast creation of output spectra by convolution of the input continuum with the corresponding Green's functions. An XSPEC implementation of this model and an initial set of tables will soon be available to the community. While this model follows similar physical assumptions and the same Green's functions strategy as previous work, the Monte-Carlo code has been completely redone from scratch. The new code not only provides new geometries, the possibility to trace every single photon during its way through the medium and the flexibility to introduce new simulation parameters easily as necessary for a feasible comparison to data but also yields corrected scattering profiles.
\end{abstract}

An INTEGRAL view of the high-energy sky (the first 10 years) - 9th INTEGRAL Workshop and celebration of the 10th anniversary of the launch

15-19 October 2012

Bibliotheque Nationale de France, Paris, France

\footnotetext{
* Speaker.
} 


\section{Introduction}

The X-ray binary systems considered here consist of a neutron star with a strong magnetic field and an optical companion donating matter to the former. The inflowing matter couples to the magnetic field of the neutron star, forming accretion columns. X-ray continuum photons are produced within the column via various processes such as cyclotron and blackbody radiation. Their further interaction with the accreted and magnetically quantized plasma electrons imprints cyclotron resonant scattering features on the spectrum. While the plasma electrons have a thermal momentum distribution parallel to the magnetic field, the electrons' momenta perpendicular to the field are quantized [1]. The corresponding energy states, the so called Landau levels, are proportional to the magnetic field strength. Therefore the mechanisms of absorption, reemission, and scattering of photons resonant in the rest frame of the involved electron form absorption-like features in the spectra allowing for a direct measurement of the neutron star's magnetic field.

\section{The Monte-Carlo approach}

Due to the coupling of the scattering photon's angle and energy to the electron momentum the exact shape of the cyclotron line can not be determined analytically. An alternative is using the Monte-Carlo approach [1] where many photons are propagated through a well defined medium. The interactions with electrons of a certain parallel temperature, $T$, in the vicinity of a strong magnetic field, $B,\left(\sim 10^{12}\right.$ Gauss $)$ alter the angle and energy of scattered photons. Photons escaping the medium are collected and individually stored in photon tables. Thus, they can be binned arbitrarily in the post-calculation phase.

Figure 1 shows the complete propagation process including photon spawning occurring whenever an electron is excited to a higher Landau level. Many probability distributions have to be calculated for the sampling of the scattering parameters. For example the angular dependent scattering cross sections are utilized for the sampling of the scattering angle. For properties of the transitions of electrons to lower Landau levels relativistic decay rates consistent with the ones derived by [2] have been used. By injecting monoenergetic photons of a certain input energy, the response of a medium defined by its geometry, magnetic field and temperature is simulated and stored in Green's function tables following the approach from [3]. These tables are used in the fitting process to calculate the emergent spectrum for an arbitrary input continuum by convolving it with the Green's functions.

\section{Mean free path interpolation tables}

The cyclotron scattering cross sections used in this work are based on R. Sina's PhD thesis [4]. They depend on the energy and angle of the scattering photon in the scattering electrons rest frame and therefore due to relativity also on the electron momentum. For the calculation of the mean free path the scattering cross sections are averaged over a relativistic distribution of electron momenta $f_{\mathrm{e}}(p)$. This involves an integration over all possible electron momenta:

$$
\langle\sigma(k, \mu)\rangle_{f_{\mathrm{e}}}=\int_{-\infty}^{\infty} \mathrm{d} p f_{\mathrm{e}}(p)(1-\mu \beta) \sigma_{\mathrm{rf}}\left(k_{\mathrm{rf}}, \mu_{\mathrm{rf}}\right)
$$




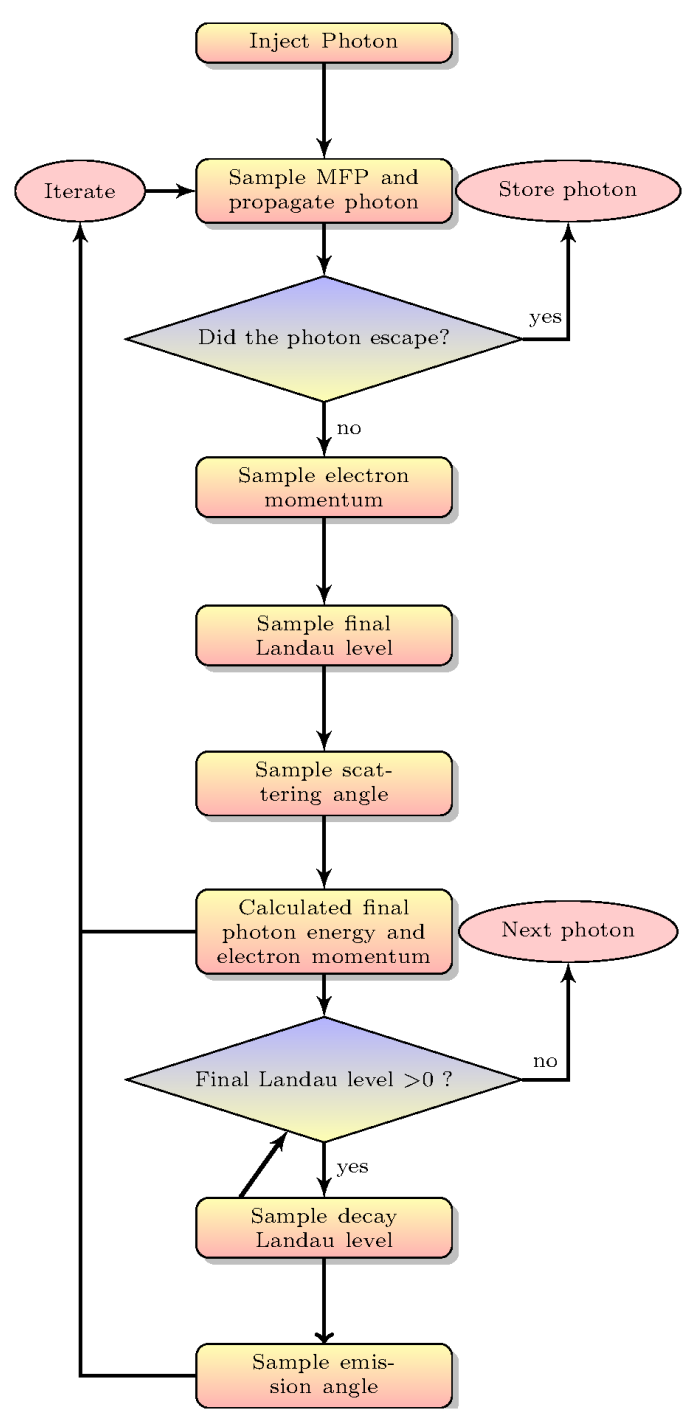

- Photon injection: The software provides different photon source types (point, plane, line). Arbitrary source dimensions and spectral properties can be easily configured.

- Propagation: The mean free path (MFP) can be calculated or interpolated. The medium can have the geometrical shape of either filled or hollow cylinders specified by their optical depths $\tau_{\|}$and $\tau_{\perp}$ parallel and perpendicular to the magnetic field.

- Sampling: The electron momentum, final Landau level and scattering angle are sampled using the corresponding distribution functions.

- Kinematics: Conservation of energy and momentum yield the final photon energy and angle.

- Photon spawning: If an electron is excited to a Landau level above the ground state it emits a photon almost immediately. The deexcitation takes place preferentially to the next lower Landau level effectively emitting a resonant photon within the fundamental line. Further deexcitation steps follow until the ground state is reached.

Figure 1: Flowchart of the Monte-Carlo process

with the cosine of the photon's angle to the magnetic field, $\mu=\cos (\vartheta)$, its energy, $k$, and the electron's velocity $\beta=v / c$. Rest frame identities are marked with subscripts. The resulting profile corresponds to the inverse mean free path $\lambda(k, \mu)=1 /\langle\sigma(k, \mu)\rangle_{f_{\mathrm{e}}}$. The distance $\Delta \tau$ the photon actually travels before scattering takes place can be sampled by assigning a random number $\mathrm{Rn}$ to the normalized primitive of its probability $W(x \rightarrow x+\Delta \tau)=\exp (-\Delta \tau / \lambda(k, \mu))$ which results in

$$
\Delta \tau=-\lambda(k, \mu) \ln (\mathrm{Rn})
$$

The integrals necessary to calculate the mean free paths involve the calculation of many cross sections. This time consuming calculation can be replaced by an interpolation from previously calculated tables. The averaged cross sections are evaluated on an energy grid which is refined adaptively until the interpolation does reproduce the calculated values within the desired accuracy. A similar approach is used to refine the angular grid. 


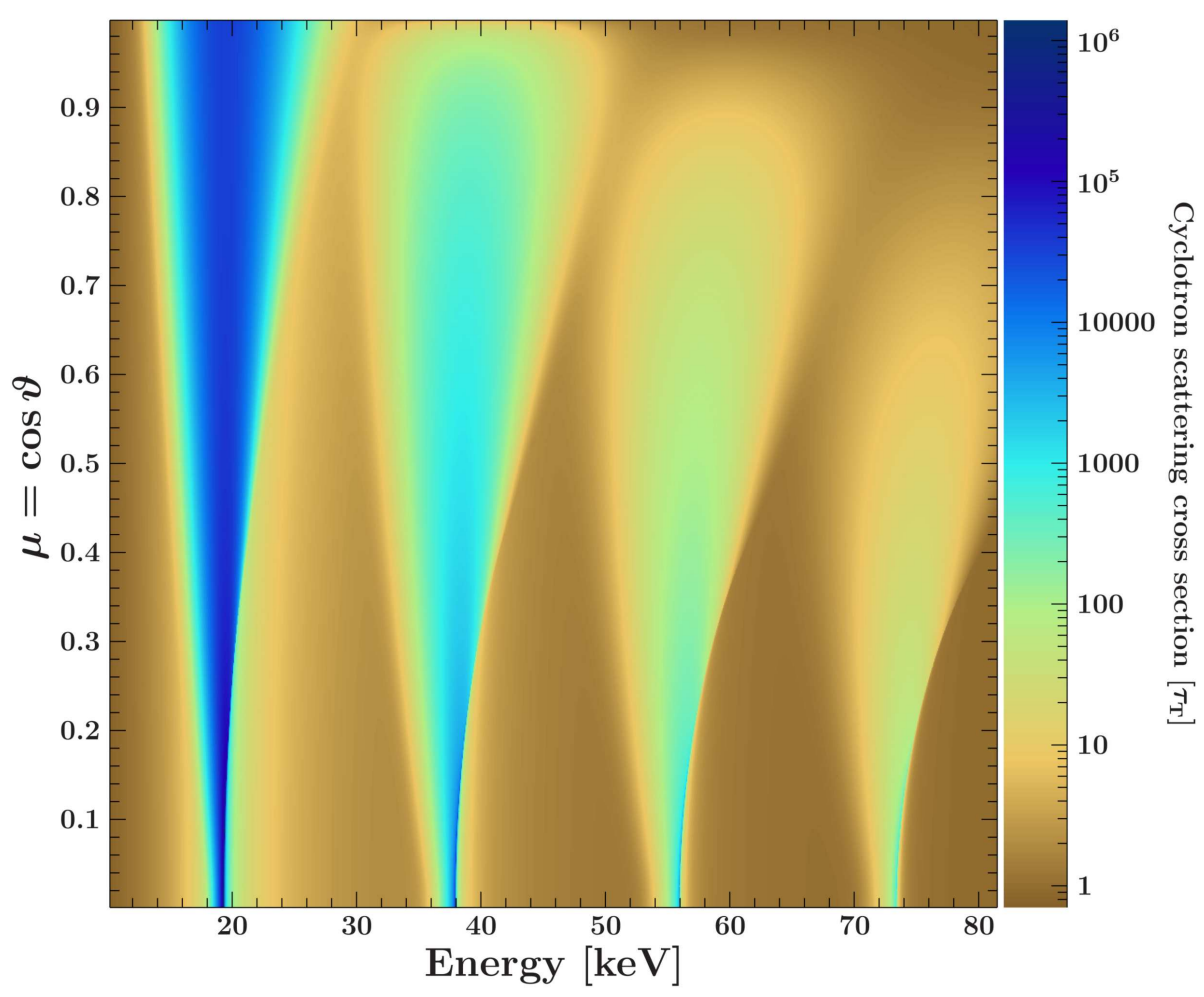

Figure 2: Illustration of the mean free path interpolation tables showing the thermally averaged scattering cross sections for a magnetic field strength of $B=0.0385 B_{\text {crit }}$ and a temperature of $T=5 \mathrm{keV}$.

This way enough points are evaluated close to the resonances while without the resonances a coarse grained grid is used to save computing time. Figure 2 depicts such an interpolation table. The colors correspond to the total cyclotron scattering cross sections averaged over a relativistic electron momentum distribution of temperature $T=5 \mathrm{keV}$. The magnetic field $B=0.0385 B_{\text {crit }}$ is in the validity range of the model which goes from $\sim 0.01 B_{\text {crit }}$ to $\sim 0.11 B_{\text {crit }}$. At higher magnetic fields other processes have to be taken into account (see [4]). The actual interpolation tables also contain all the partial integrals which are used for the sampling of the scattering electron's momentum.

\section{Generalizing the accretion column's geometry}

Our model extends the simple slab and cylinder geometries of the line-forming region used in previous work $[1,3]$ to the much more flexible concept of stacked hollow cylinders consisting of generalized cylinders which can be stacked on top of, or even around other cylinders. The original slab geometries correspond to flat cylinders with a certain optical depth parallel to the magnetic field and an infinite radius. All optical depths are given in units of the Thomson optical depth $\tau_{\mathrm{Th}}$. The cylinders are illuminated by a source plane which in the case of slab 1-1 geometry is placed in the middle of the cylinder. Slab 1-0 geometry corresponds to a bottom illuminated 


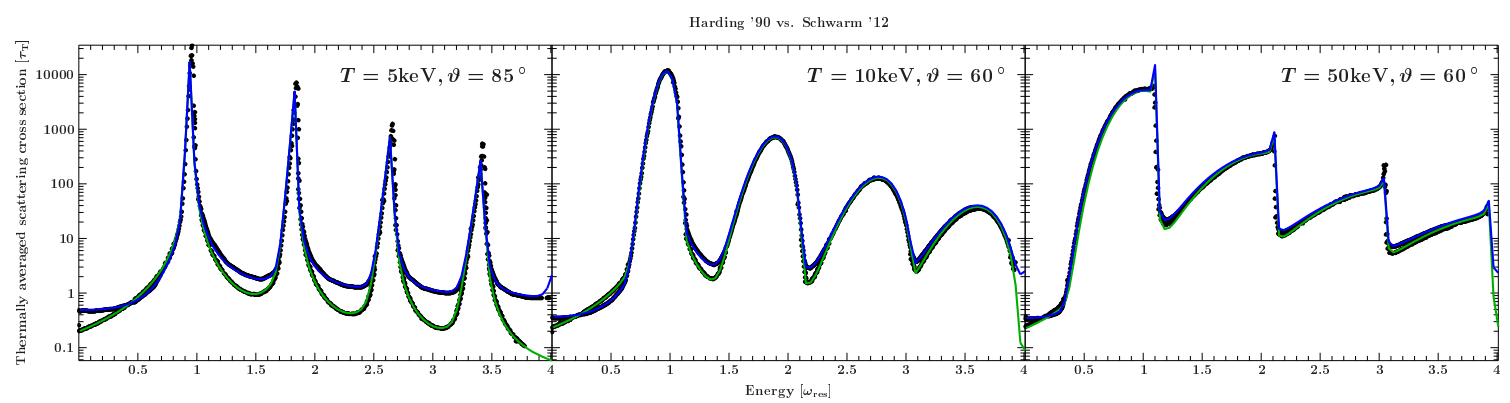

Figure 3: Inverse mean free paths (line profiles) in comparison to the ones from [5] for a magnetic field of $0.1 B_{\text {crit. }}$. Different temperatures $T$ and angles $\vartheta$ to the magnetic field are shown.

cylinder. Using stacked hollow cylinders, radial gradients are possible as well as gradients in height providing sufficient complexity to model realistic accretion columns. In principal all kinds of cylindrical geometries can be modeled this way with available computing power being the only limitation. Comparing CRSF spectra which are simulated using only one cylinder but with different combinations of optical depths parallel and perpendicular to the magnetic field to observational data may provide inside into whether the observed accretion column bears more analogy to slabor cylinder-like geometries.

\section{Impact of corrected scattering profiles}

Our new MC code is following a very similar physical scheme as the code by [1], a revised version of which we have previously used as basis and for the development of an XSPEC fitting model for the analysis of cyclotron lines [3]. We found the code by [1], however, to numerically overestimate the scattering cross section profiles which theoretically should but in fact do not agree with previously published scattering profiles [5]. This led to an overestimation of the depths of the CRSFs deviating considerably also from results of different numerical implementations of CRSF models like, e.g., [6], and originally motivated programming the new MC code, presented here.

Figure 5 shows a comparison of our simulated spectra to [1] for different optical depths and reveals the differences in line depths originating from the usage of corrected profile functions. Our profiles now agree perfectly with [5] (see Figure 3). Figure 5 shows a comparison of our spectra (red/green) to [6] (black). Slab 1-0 (green) geometry is shown as well as slab 1-1 geometry (red). Both spectra, though produced with completely independent simulations, now agree very well.

\section{Outlook}

The comparison to data has just begun. Up to now cyclotron lines have mainly been fitted using multiple Gaussian absorption lines. With our model multiple cyclotron lines can be fitted simultaneously using a physically reasonable model. We plan to merge the model for the formation of CRSFs in arbitrary continua, presented here, with the analytic approach by Becker \& Wolff [7] to a physical calculation of X-ray pulsar continua. This will lead to a hybrid model which can be used to fit the continuum and the lines at the same time. The inclusion of general relativistic effects and rotating two pole geometry in the future will make the model even more realistic since we expect these processes to significantly influence the angular mixing of the emergent radiation. This 

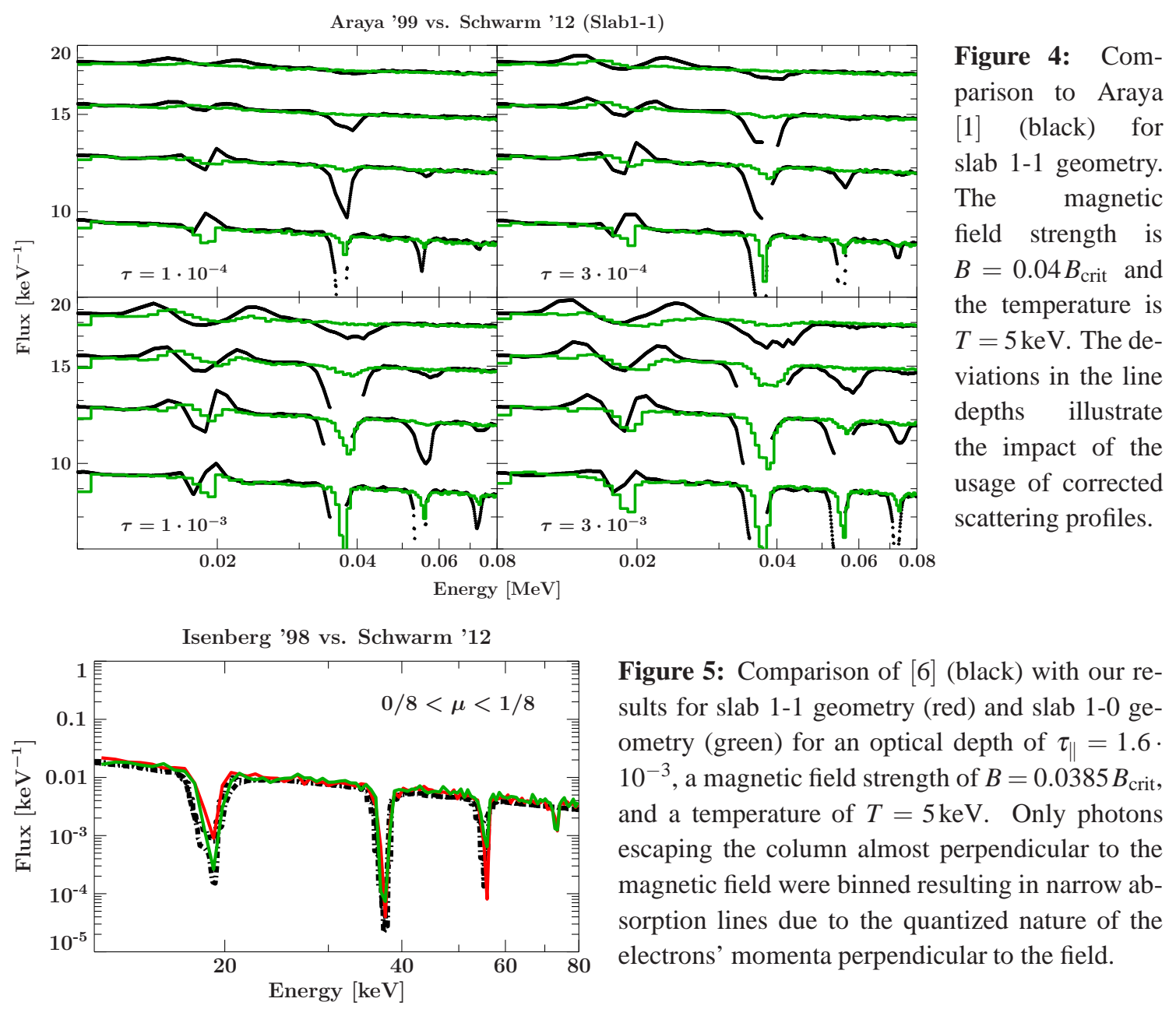

Figure 5: Comparison of [6] (black) with our results for slab 1-1 geometry (red) and slab 1-0 geometry (green) for an optical depth of $\tau_{\|}=1.6$. $10^{-3}$, a magnetic field strength of $B=0.0385 B_{\text {crit }}$, and a temperature of $T=5 \mathrm{keV}$. Only photons escaping the column almost perpendicular to the magnetic field were binned resulting in narrow absorption lines due to the quantized nature of the electrons' momenta perpendicular to the field.

will further constrain the physical parameters in the accretion column by establishing a connection between spectral and timing information and for the first time enable us to perform a fully consistent analysis of phase-resolved spectroscopic data with a physical model.

This work has been supported in part by the Bundesministerium für Wirtschaft und Technologie under Deutsches Zentrum für Luft- und Raumfahrt grant number 50OR0905.

\section{References}

[1] Araya, R. A., \& Harding, A. K. 1999, ApJ, 517, 334

[2] Herold, H., Ruder, H., \& Wunner, G. 1982, A\&A, 115, 90

[3] Schönherr, G., Wilms, J., Kretschmar, P., Kreykenbohm, I., Santangelo, A., Rothschild, R. E., Coburn, W., \& Staubert, R. 2007, A\&A, 472, 353

[4] Sina, R. 1996, PhD thesis, Univ. Maryland

[5] Harding, A. K. \& Daugherty, J. K. 1991, ApJ, 374, 687

[6] Isenberg, M., Lamb, D. Q., \& Wang, J. C. L. 1998, ApJ, 505, 688

[7] Becker, P. A., \& Wolff, M. T. 2007, ApJ, 654, 435 